\title{
Atomic Form Factor Calculations of S-states of Helium
}

\author{
Saïdou Diallo*, Ibrahima Gueye Faye, Louis Gomis, Moustapha Sadibou Tall, Ismaïla Diédhiou
}

Department of Physics, Faculty of Sciences, University Cheikh Anta Diop, Dakar, Senegal

Email address:

saidou1.diallo@ucad.edu.sn (S. Diallo)

*Corresponding author

\section{To cite this article:}

Saïdou Diallo, Ibrahima Gueye Faye, Louis Gomis, Moustapha Sadibou Tall, Ismaïla Diédhiou. Atomic Form Factor Calculations of S-states of Helium. American Journal of Modern Physics. Vol. 8, No. 4, 2019, pp. 66-71. doi: 10.11648/j.ajmp.20190804.12

Received: September 12, 2019; Accepted: October 4, 2019; Published: October 15, 2019

\begin{abstract}
Variational calculations of the helium atom states are performed using highly compact 26-parameter correlated Hylleraas-type wave functions. These correlated wave functions used here yield an accurate expectation energy values for helium ground and two first excited states. A correlated wave function consists of a generalized exponential expansion in order to take care of the correlation effects due to N-corps interactions. The parameters introduced in our model are determined numerically by minimization of the total atomic energy of each electronic configuration. We have calculated all integrals analytically before dealing with numerical evaluation. The $1 S^{2} 1{ }^{1} S$ and $1 S 2 S 2^{1,3} S$ states energies, charge distributions and scattering atomic form factors are reported. The present work shows high degree of accuracy even with relative number terms in the trial Hylleraas wave functions definition. The results presented here, indicate that the highly compact twenty-six variational parameters model will have the quantitative and qualitative applicability for the study of electronic correlation. The correlated wave functions are used to calculate the atomic form factor for the diffusion of electrons by the helium atom. The atomic form factor is evaluated as the Fourier transform of the electron density distribution of an atom or ion, which is calculated from theoretical correlated wave functions for free atoms. Finally, suggestions are made as to the way the atomic form factor of the helium atom may be approximated by a sum of Gaussians for efficiency use.
\end{abstract}

Keywords: Helium, Variational Method, Correlation Effect, Charge Distribution, Scattering

\section{Introduction}

Several theories and methods of calculations have been elaborated these last years to calculate energy levels and electronic charge distribution of atoms and molecules. The method of the self-consistent field proposed in 1927 by D. Hartree is one of the best resolution processes of the quantum chemistry problems. The stationary Schrödinger equation can be solved analytically in a very restricted number of case (atomic hydrogen, harmonic oscillator, etc.) Most problems of the chemistry and quantum mechanics are solved with the help of approximate methods. In many cases, variational method enables us to solve much more efficiently the Schrödinger equation.

The problem of bound states of two-electron systems has a long history an attracts continuing interest. The variational principle of solving this problem is largely used. In order to have accurate final wave function and energy, an adequate trial function is chosen and a large number of variational parameters must be introduce [1]. The number of parameters increases with the system size up to several hundreds and the optimization requires efficient strategies [2]. Several kinds of trial functions are used to deal with two-electron atomic or ion systems [3-6]. A comparative study of two-electron systems with different potentials is done by authors of Ref. [7]. They discuss the limits of validity of the correlation hypothesis. Usually, the method consists of the product of two factors wave functions. The first term includes the electron-nucleus interactions. The second term takes into account both the electron-electron interaction and the correlation between all the parts of the system elements. This second terms can be constructed by a sum with a large number of parameters or by a specific mathematical function. The Hartree-Fock method, the multiconfiguration HartreeFock method and the Ritz method both apply the variational method. The variational method is sometimes, combined with other methods to investigate the bound states of twoelectron system [8]. Highly correlated wave functions can be obtained by including more terms in the polynomial 
representation of the correlation function [9-12]. Alternative correlated wave functions are proposed with few terms in the expansion of the correlation [13-15].

As illustration, we will discuss two electron systems; this includes helium and helium like systems. These are the simplest systems in which we can study correlation, but they do exhibit many interesting challenges of complex atoms. The $1^{1} S$ and $2^{1,3} S$ states energies of the helium atom are calculated by using Hylleraas basis wave functions that introduces the coordinates $(s, t, u)$. The principal reason of introducing these new variables was the volume integral reduction from six to three integrals. A screening parameter has been introduced in our trial wave functions that can be seen as an effective nuclear charge of the atom. The main purpose of the present paper is to extend the calculation of the ground and two first excited states energy of helium. In the other hand, the calculation of charge distribution is important for the determination of scattering atomic form factor. The atomic form factor is the scattering contribution from a single, isolated atom. Generally, it describes the shape of a scattering object. More specifically, it is the Fourier transform of the atom's spatial distribution. An accurate calculation of wave function is very important to determine the spatial distribution that governs the shape of the atomic form factor. In the most general case of an arbitrary distribution of scattering density, the form factor is computed by integrating over all space.

The paper is structured as follows. In section 2 we construct the twenty-six parameters variational wave function. In this section, we have also established all the analytical expressions involved in the calculation and minimization of the total energy of the system and the electronic density of each configuration respectively. We derive in section 3, the explicit expression of the scattering atomic form factor. The analytical expressions are obtained with the spherical symmetry density. The binomial expansion is used to evaluate the radial integrals where it's needed. We compare our results with some previous calculations done by other authors in section 4 .

\section{Variational Calculations}

The Schrödinger equation for the helium atom, or the positive ions or the negative hydrogen ion of the isoelectronic sequence is

$$
\Psi=E \Psi
$$

Where the Hamiltonian $H$ is given by

$$
H=-\frac{1}{2} \nabla_{1}^{2}-\frac{1}{2} \nabla_{2}^{2}-\frac{Z}{r_{1}}-\frac{Z}{r_{2}}+\frac{1}{r_{12}}
$$

where $r_{1}, r_{2}$ are the coordinates of the two electrons; $r_{12}$ is the distance between them, $Z=2$ the charge atomic number, $\Psi$ and $E$ the eigenfunction and eigenenergy of the system. The complete description of the S-states requires only three independent coordinates, $r_{1}, r_{2}$ and $r_{12}$ [16]. The effects of correlation are globally introduced in the wave functions by inter-electronic repulsion potential $\frac{1}{r_{12}}$. Variational methods for solving the Schrödinger equation (1) rely on a reformulation of the eigenvalue problem. Solving the Schrödinger equation for bound states is equivalent to finding functions $\Psi$ that leave the energy functional

$$
[\Psi]=\frac{\langle\Psi|H| \Psi\rangle}{\langle\Psi \mid \Psi\rangle}
$$

Which is recognized as the expectation value of the energy for a stationary state $\Psi$ satisfying the boundary conditions. In addition to the boundary conditions, the variation must also have the expected integrability, continuity and differentiability properties. With the natural Hylleraas coordinates $s=r_{1}+r_{2}, t=r_{1}-r_{2}, u=\left|r_{1}-r_{2}\right|$. We see that the variables $s$ and $u$ are always positives but the variable $t$ can take both the positive or negative values. The integrals in equation (3) will contain two parts. The contribution to the integral from $(-t)$ is identical with that from $t$. In this condition, we multiplied the volume element by a factor of 2 . The resulting volume element is now found to be in the following form

$$
d V=2 \pi^{2}\left(s^{2}-t^{2}\right) u d s d t d u
$$

The limits of integration become

$$
0 \leq t \leq u \leq s \leq+\infty
$$

The form of the wave functions used is as follows [17]

$$
\Psi(s, t, u)=\sum_{i=0}^{n} C_{i} \Phi_{i}(s, t, u)
$$

From the variational principle, one obtains the matrix eigenvalue problem:

$$
(H-E N) C=0
$$

The Hamiltonian and overlap matrix are represented by $H$ and $N$ respectively. The coefficients $C_{i}$ are elements of the $\mathrm{C}$ matrix. The secular equation is:

$$
\left|\begin{array}{cccc}
H_{11}-\varepsilon N_{11} & H_{12}-\varepsilon N_{12} & \cdots & H_{1 n}-\varepsilon N_{1 n} \\
H_{21}-\varepsilon N_{21} & H_{22}-\varepsilon N_{22} & \cdots & H_{2 n}-\varepsilon N_{2 n} \\
\vdots & \vdots & \vdots & \\
H_{n 1}-\varepsilon N_{n 1} & H_{n 2}-\varepsilon N_{n 2} & \cdots & H_{n n}-\varepsilon N_{n n}
\end{array}\right|=0
$$

Where $\varepsilon$ is the eigenvalue matrix and the matrix elements $H_{i j}=T_{i j}+V_{i j}$. The kinetic energy matrix elements of the Hamiltonian are defined by:

$$
T_{i j}=\int_{0}^{\infty} d s \int_{0}^{s} d u \int_{0}^{u} d t\left(A_{0}+A_{1}+A_{2}\right)
$$

Where

$$
\begin{aligned}
& A_{0}=u\left(s^{2}-t^{2}\right)\left[\frac{\partial \Phi_{i}}{\partial s} \frac{\partial \Phi_{j}}{\partial s}+\frac{\partial \Phi_{i}}{\partial t} \frac{\partial \Phi_{j}}{\partial t}+\frac{\partial \Phi_{i}}{\partial u} \frac{\partial \Phi_{j}}{\partial u}\right] \\
& A_{1}=s\left(u^{2}-t^{2}\right)\left[\frac{\partial \Phi_{i}}{\partial u} \frac{\partial \Phi_{j}}{\partial s}+\frac{\partial \Phi_{j}}{\partial u} \frac{\partial \Phi_{i}}{\partial s}\right] \\
& A_{2}=t\left(s^{2}-u^{2}\right)\left[\frac{\partial \Phi_{i}}{\partial u} \frac{\partial \Phi_{j}}{\partial t}+\frac{\partial \Phi_{j}}{\partial u} \frac{\partial \Phi_{i}}{\partial t}\right]
\end{aligned}
$$

and the potential matrix elements $V_{i j}$ are expressed as 


$$
V_{i j}=\int_{0}^{\infty} d s \int_{0}^{s} d u \int_{0}^{u} d t\left[4 Z s u+t^{2}-s^{2}\right] \Phi_{i} \Phi_{j}
$$

The overlap matrix elements are given as:

$$
N_{i j}=\int_{0}^{\infty} d s \int_{0}^{s} d u \int_{0}^{u} d t u\left(s^{2}-t^{2}\right) \Phi_{i} \Phi_{j}
$$

It is important to remark, the small difference introduced in our two equations (11) and (12). The factor 2 that appears in equation (32.17) in the reference [18], is replaced by a derivative sum. We assume in this work, that the trial function $\Phi_{i}(s, t, u)$ is expressed as

$$
\Phi_{i}(s, t, u)=e^{-\alpha s / 2} s^{p_{i}} t^{q_{i}} u^{r_{i}}
$$

Where $\alpha$ is a nonlinear variational parameter. The $p_{i}, q_{i}$ and $r_{i}$ are integers that define each term of the trial function. In order to check our calculations, we use a few terms in the trial function definition like $p=[0,0,0,0,2,1], q=$ $[0,0,2,0,0,0]$ and $r=[0,1,0,2,0,1]$. With these values, we obtain the ground state energy $E=-2.9033$ u.a and the nonlinear parameter that represent the screening factor is $\alpha=3.78$. The corresponding wave function can be written using equations (6) and (15) as

$$
\begin{aligned}
\Psi(s, t, u)= & e^{-1.89 s}\left(-6.1037-1.8970 u-0.9212 t^{2}+\right. \\
& \left.0.4466 u^{2}-0.0513 s^{2}-0.5811 s u\right)
\end{aligned}
$$

The wave function $\Psi$ of (6) must be multiplied by $\mathcal{N}$ in order to be normalized

$$
\mathcal{N}=\frac{1}{\sqrt{\langle\Psi \mid \Psi\rangle}} ;\langle\Psi \mid \Psi\rangle=\sum_{i, j}^{n} C_{i} C_{j} \Phi_{i} \Phi_{j}
$$

One can determine the charge distribution by

$$
\rho\left(r_{1}\right)=\int \Psi^{2} d r_{2}
$$

To deal with this integral, we consider the triangle formed by the three vectors $r_{1}, r_{2}, r_{12}$ and pass the $z-$ axis by rotation along $r_{1}$, which is taken for a moment as a constant. The angles transformation give $\theta_{2} \rightarrow \theta_{12}$ and $\Phi_{2} \rightarrow \Phi_{12}$. This rotation allows us to write the volume element of electron 2 as $d r_{2}=r_{2}^{2} d r_{2} d \Phi_{12} \sin \theta_{12} d \theta_{12}$. Then equation (16) can be put in the following form

$$
\rho\left(r_{1}\right)=\int_{0}^{\infty} r_{2}^{2} d r_{2} \int_{0}^{2 \pi} d \Phi_{12} \int_{0}^{\pi} \Psi^{2} \sin \theta_{12} d \theta_{12}
$$

As $\Phi_{12}$ is an independent variable, we first integrate over $\Phi_{12}$ and we get a factor $2 \pi$. The angle $\theta_{12}$ is related to the variable $r_{12}$ by:

$$
r_{12}^{2}=r_{1}^{2}+r_{2}^{2}-2 r_{1} r_{2} \cos \theta_{12}
$$

By differentiating on both sides of this equation, we obtain the needed relation as

$$
\sin \theta_{12} d \theta_{12}=\frac{r_{12}}{r_{1} r_{2}} d r_{12}
$$

Substituting the relation (21) into (19), and rewriting properly the integration domain, we have:

$$
r_{1} \rho\left(r_{1}\right)=2 \pi \int_{0}^{\infty} r_{2} d r_{2} \int_{\left|r_{1}-r_{2}\right|}^{r_{1}+r_{2}} \Psi^{2} r_{12} d r_{12}
$$

The integration over the $r_{12}$ coordinate is possible. There are two regions of integration: $r_{1}>r_{2}$ and $r_{1}<r_{2}$. So we can split the domain integration into two terms. In this way the charge distribution is written as

$$
\begin{array}{r}
\rho\left(r_{1}\right)=\rho_{a}\left(r_{1}\right)+\rho_{b}\left(r_{1}\right), \text { where we have set } \\
\rho_{a}\left(r_{1}\right)=\frac{2 \pi}{r_{1}} \int_{0}^{r_{1}} r_{2} d r_{2} \int_{r_{1}-r_{2}}^{r_{1}+r_{2}} \Psi^{2} r_{12} d r_{12} \\
\rho_{b}\left(r_{1}\right)=\frac{2 \pi}{r_{1}} \int_{r_{1}}^{\infty} r_{2} d r_{2} \int_{r_{2}-r_{1}}^{r_{1}+r_{2}} \Psi^{2} r_{12} d r_{12}
\end{array}
$$

To evaluate the two integrals in equations (23) and (24), the binomial theorem will be useful.

$$
\rho_{a}\left(r_{1}\right)=\frac{1}{\pi} \sum_{i}^{n} \sum_{j}^{n} \frac{C_{i} C_{j} e^{-\alpha r_{1}}}{r_{i}+r_{j}+2}\left(D_{1}-D_{2}\right)
$$

With

$$
D_{1}=\sum_{k_{1}}^{n_{1}} \sum_{k_{2}}^{n_{2}}(-1)^{k_{2}}\left(\begin{array}{l}
n_{1} \\
k_{1}
\end{array}\right)\left(\begin{array}{l}
n_{2} \\
k_{2}
\end{array}\right) \frac{l_{1} ! r_{1}^{m-l_{1}}}{\alpha^{l_{1}+1}} S\left(l_{1}\right)
$$

The function $S(l)$ is defined later in equation (28)

$$
D_{2}=\sum_{k_{3}}^{n_{3}} \sum_{k_{4}}^{n_{4}}(-1)^{k_{4}}\left(\begin{array}{l}
n_{3} \\
k_{3}
\end{array}\right)\left(\begin{array}{l}
n_{4} \\
k_{4}
\end{array}\right) \frac{l_{2} ! r_{1}^{m-l_{2}}}{\alpha^{l_{2}+1}} S\left(l_{2}\right)
$$

where we have $n_{1}=p_{i}+p_{j}+r_{i}+r_{j}+2, n_{2}=q_{i}+q_{j}$, $n_{3}=p_{i}+p_{j}, n_{4}=q_{i}+q_{j}+r_{i}+r_{j}+2, l_{1}=k_{1}+k_{2}+$ $1, l_{2}=k_{3}+k_{4}+1, \quad m=n_{1}+n_{2}+1$, and $\left(\begin{array}{l}n \\ k\end{array}\right)$ are the binomial factors. The quantity $S(l)$ is define as

$$
S(l)=1-e^{-\alpha r_{1}} \sum_{k=0}^{l} \frac{\left(\alpha r_{1}\right)^{k}}{k !}
$$

The same procedure is used to derive

$$
\rho_{b}\left(r_{1}\right)=\frac{1}{\pi} \sum_{i}^{n} \sum_{j}^{n} \frac{C_{i} C_{j} e^{-\alpha r_{1}}}{r_{i}+r_{j}+2}\left(D_{1}-(-1)^{s} D_{2}\right)
$$

with $s=r_{i}+r_{j}$. In equation (27), everything is kept as in (23) except $S(l) \rightarrow 1-S(l)$. By using the Hylleraas coordinates, on can easily evaluate the normalization constant as:

$$
\langle\Psi \mid \Psi\rangle=\sum_{i, j}^{n} C_{i} C_{j} C_{0} \frac{(m+2) !}{\alpha^{m+3}}
$$

Where

$$
C_{0}=\frac{1}{\left(n_{2}+1\right)\left(n_{4}+1\right)}-\frac{1}{\left(n_{2}+2\right)\left(n_{4}+3\right)}
$$

The numbers $n_{2}, n_{4}$ and $m$ are defined above. The total spherical charge distribution is obtained as

$$
\rho(r)=2 \rho\left(r_{1}=r\right)
$$

\section{Atomic Form Factor}

The scattering atomic form factor obtained from first Born approximation has the following form:

$$
T(q)=\int \rho(r) \exp (i q \cdot r) d^{3} r
$$

Thus $T(q)$ is proportional to Fourier transform of the 
electronic density $\rho(r)$. For the spherical symmetry density, we have:

$$
T(q)=4 \pi \int_{0}^{\infty} r^{2} \rho(r) \frac{\sin q r}{q r} d r
$$

where $q=2 k \sin (\theta / 2)$ is the transfer momentum to the atomic system. $k$ and $\theta$ are respectively the wave number and scattered angle of the electron. We can remark, when the momentum tends to zero, the limit of $T(q)$ is

$$
\lim _{q \rightarrow 0} T(q)=\int_{0}^{\infty} r^{2} \rho(r) d r=Z
$$

One can evaluate without any difficulties the atomic form factor integrals with the explicit form of the charge distribution of equation (32). After calculations, we find

$$
2 i q T(q)=T_{1}(q)-T_{1}(-q)+T_{2}(q)-T_{2}(-q)
$$

We remind that $i$ is the complex symbol.

$$
\begin{gathered}
T_{1}(q)=\sum_{i}^{n} \sum_{j}^{n} \frac{c_{i} c_{j}}{r_{i}+r_{j}+2}\left\{T_{11}(q)-T_{12}(q)\right\} \\
T_{2}(q)=\sum_{i}^{n} \sum_{j}^{n} \frac{c_{i} c_{j}}{r_{i}+r_{j}+2}\left\{T_{21}(q)-(-1)^{s} T_{22}(q)\right\}
\end{gathered}
$$

With

$$
\begin{aligned}
& T_{11}(q)=\sum_{k_{1}}^{n_{1}} \sum_{k_{2}}^{n_{2}}(-1)^{k_{2}}\left(\begin{array}{l}
n_{1} \\
k_{1}
\end{array}\right)\left(\begin{array}{l}
n_{2} \\
k_{2}
\end{array}\right) \frac{l_{1} ! r_{1}^{m-l_{1}}}{\alpha^{l_{1}+1}} R\left(d_{1}, l_{1}\right) \\
& T_{12}(q)=\sum_{k_{3}}^{n_{3}} \sum_{k_{4}}^{n_{4}}(-1)^{k_{4}}\left(\begin{array}{l}
n_{3} \\
k_{3}
\end{array}\right)\left(\begin{array}{l}
n_{4} \\
k_{4}
\end{array}\right) \frac{l_{2} ! r_{1}^{m-l_{2}}}{\alpha^{l_{2}+1}} R\left(d_{2}, l_{2}\right)
\end{aligned}
$$

And

$$
R(d, l)=\frac{d !}{(\alpha-i q)^{d+1}}-\sum_{k=0}^{l} \frac{(d+k) ! \alpha^{k}}{k !(2 \alpha-i q)^{d+k+1}}
$$

with $d_{1}=n_{1}+n_{2}+1-l_{1}, d_{2}=n_{1}+n_{2}+1-l_{2} . T_{21}$ and $T_{22}$ have respectively the same expression as $T_{11}$ and $T_{12}$ with here

$$
R(d, l)=\sum_{k=0}^{l} \frac{(d+k) ! \alpha^{k}}{k !(2 \alpha-i q)^{d+k+1}}
$$

The expressions derived here in order to evaluate the atomic form factor are very complicate to deal with. For future applications of this work, the atomic form factor is well approximated, in the range of scattering momentum transfer between $0 \leq q \leq 10$, by a sum of Gaussians of the form:

$$
T(q)=\sum_{i=1}^{6} a_{i} \exp \left(-b_{i} q^{2}\right)+a_{0}
$$

Where the values of the constants $a_{i}, b_{i}$ and $a_{0}$ are tabulated below in table 2 . The equation (43) is more tractable to deal with the atomic form factor than equation (36).

\section{Results and Discussions}

We compare in table 1 calculated energies obtained with our fifteen terms wave function with other theoretical model. Our calculations are accurate within an absolute error $\Delta E=E([19]-\mathrm{E}($ present work $)): 0.67610^{-6}$ for $1^{1} S$,
$699.6410^{-6}$ for $2^{1} S$ and $35.9310^{-6}$ for $2^{3} S$.

Table 1. The nonrelativistic energies for the $1^{1} S, 2^{1} S$ and $2^{3} S$ states of He atom. Bold numbers are from the present work, italic numbers from reference [20] and normal numbers are from reference [19].

\begin{tabular}{lll}
\hline States & Basis & -E (u.a) \\
\hline \multirow{3}{*}{$1^{1} S$} & 50 & 2.903723701 \\
& 700 & 2.90372437700 \\
& 1400 & 2.903724377034119596 \\
$2^{1} S$ & 50 & 2.145274406 \\
& 700 & 2.14597404604 \\
& 1500 & 2.14597404605441738 \\
$2^{3} S$ & 50 & 2.175193448 \\
& 500 & 2.175229378237 \\
& 1200 & 2.17522937823679130573 \\
\hline
\end{tabular}

Figures 1 and 2 show plots of the charge distribution $r^{2} \rho(r)$ versus the electron radius $r$ in atomic units (a.u) of the helium atom of states $1^{1} S$ and $2^{1,3} S$ respectively. We compare in figure 1, the hydrogenic limit and the variational Hartree-Fock models with the present Hylleraas approach. The variational HartreeFock model gives the same charge distribution as the Hylleraas model. The hydrogenic model overestimates the probability of presence in the vicinity of the maximum for state $1 \mathrm{~s} 1 \mathrm{~s}$. The curve obtained with this model decreases rapidly before being cancelled out for the large values of the radius position.

We can say that the variational Hartree-Fock model describes quite properly the atomic structure because the significant quantity is the square of the wave function that defines the electron probability presence at position $r$.

Figure 2 shows the same charge distribution for the two states $2^{1,3} S$ near the nucleus and far from the origin. At large distance it is obvious that all charge distributions functions converge to zero. This behavior is due to the asymptotic nature of the wave functions. We notice two distinct maxima that correspond to the probability of the electron's presence in orbital 1s and orbital 2s. It is logical to observe the same amplitude for the first peak that describes the orbital 1s. For the second peak, we see a discrepancy between the two curves due to the energy difference between the two states, with nevertheless the same amplitudes.

We present in figures 3 and 4 the scattering atomic form factor of electron helium collision versus the transfer momentum. In both figures, the scattering atomic form factor decreases with the increasing transfer momentum. The behavior of the plots is expected, because the energy transferred to the target becomes more import that can induce transitions or rearrangement processes when the excitation energy is reached.

In figure 4 , we remark that the scattering atomic form factor is quite the same of the two states $2^{1} S$ and $2^{3} S$. The small variation between the two states scattering atomic form factor can be explained by the similarity on their wave function form. Nevertheless, we see a slight offset of the two curves in the vicinity of $q=1$, which is explained by the probability of the presence of the second electron in the orbital $2 s$. This probability of presence varies significantly from one configuration to another. The atomic form factor tends towards zero for fairly high transfer moments. This is foreseeable because in this area moments of transfer, capture processes largely dominate. 


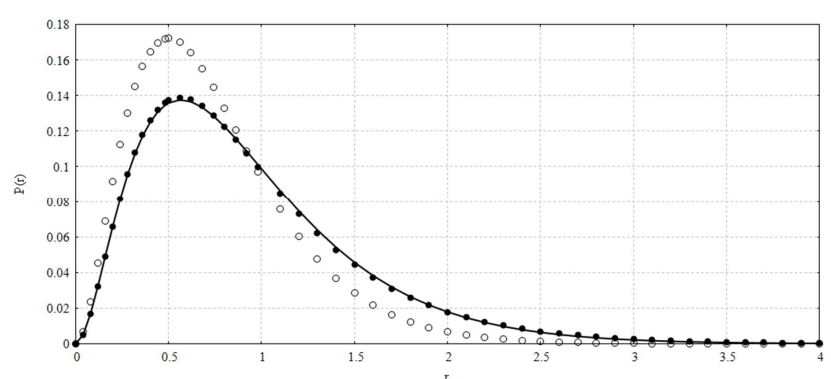

Figure 1. Plot of the ground state $1^{1} S$ charge distribution $P(r)=r^{2} \rho(r)$ from equation (32) for He. The hydrogenic and the variational Hartree-Fock charge distribution are represented respectively by filled and opened circle curve. The present Hylleraas approximation charge distribution is represented by line curve.

Table 2. The coefficients values of the approximated atomic form factor of the three states of helium atom.

\begin{tabular}{llll}
\hline Coefficients & $\mathbf{1}^{\mathbf{S}}$ & $\mathbf{2}^{\mathbf{S}} \boldsymbol{S}$ & $\mathbf{2}^{\mathbf{S}} \boldsymbol{S}$ \\
\hline$a_{0}$ & 0.00678637 & 0.00875839 & 0.0156208 \\
$a_{1}$ & 0.535535 & 0.313819 & 0.0987228 \\
$b_{1}$ & 0.269436 & 0.369448 & 0.121975 \\
$a_{2}$ & 0.0265823 & -0.360732 & 1.00182 \\
$b_{2}$ & 0.929621 & 0.586244 & 3.13333 \\
$a_{3}$ & 0.227123 & 1.12541 & 0.0753508 \\
$b_{3}$ & 0.501576 & 4.4784 & 7.89644 \\
$a_{4}$ & 0.153599 & 0.2088 & -0.0967258 \\
$b_{4}$ & 0.0211122 & 0.029995 & 0.0193181 \\
$a_{5}$ & 0.430681 & 0.521892 & 0.361228 \\
$b_{5}$ & 0.0621708 & 0.0957924 & 0.0298007 \\
$a_{6}$ & 0.619694 & 0.181254 & 0.544152 \\
$b_{6}$ & 0.13695 & 0.20935 & 0.121959 \\
\hline
\end{tabular}

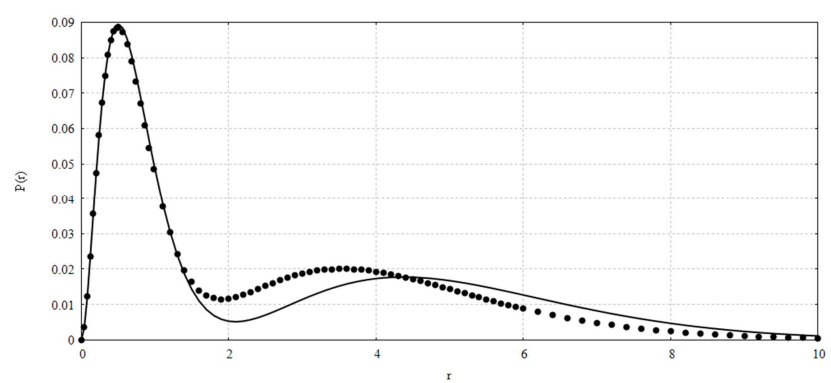

Figure 2. Plot of the first excited states $2^{1,3} S$ charge distribution $P(r)=$ $r^{2} \rho(r)$ from equation (32) for He. Line and filled circles curve are respectively for the singlet state $2^{1} S$ and triplet state $2^{3} S$.

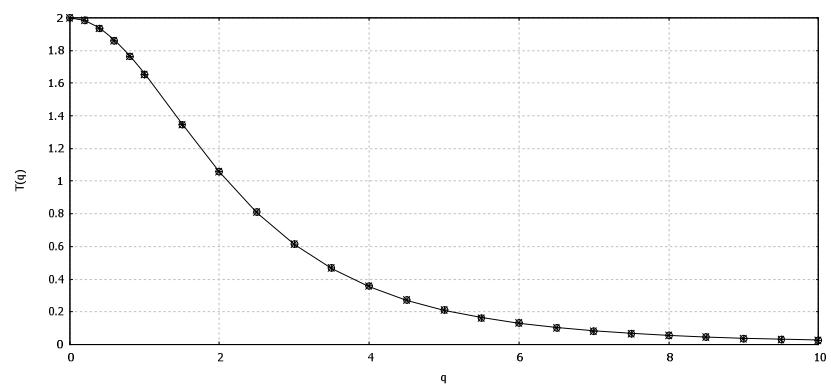

Figure 3. Plot of the ground state $1^{1} S$ atomic form factor from equation (33) for He. Line, opened and stars curves are respectively obtained by using the Hylleraas type functions, Hatree-Fock wave functions and hydrogenic limit's functions.

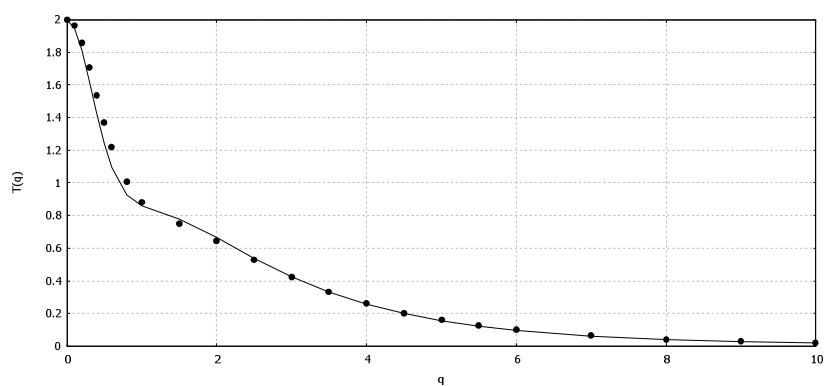

Figure 4. Plot of the first excited states $2^{1,3} S$ atomic form factor from equation (33) for He. Line and filled circles curves are respectively for the singlet state $2^{1} S$ and triplet state $2^{3} S$.

\section{Conclusion}

The energy levels for $1^{1} S$ and $2^{1,3} S$ states of helium are calculated by diagonalizing the Hamiltonian with the Hylleraas basis functions. With this method one can retrieve high excited energy levels under some degree of accuracy. We have varied the only nonlinear parameter $\alpha$ to obtain the minimum eigenenergies and corresponding wave function through the expansion coefficients $C_{i}$. We can also determine analytically the nonlinear parameter by minimizing the energy functional with $\alpha$. We find that the ground state energy is more accurate than the excite states energies. The problem of obtaining accurate wave functions for the helium atom was solved with the works of C. L. Pekeris [20], K. Frankowski [22].

These studies are very useful to understand the electronic correlations in two electron-atomic systems. The results of our work demonstrates the advantage of using four variational parameters wave functions, which have the same analytic structure for helium iso-electronic sequence. There is still some interest in trying to obtain high accuracy with smaller number of variational parameters suitable for scattering atomic form factor, generalized oscillator strength and cross sections calculations in atomic collision processes. This must involve examining the form of the correlated term which is included in the trial wave function. The choice of this trial wave function must be as efficient as possible to ensure accuracy and portability.

We show the importance to have accurate wave function in atomic collision processes namely in scattering atomic form factor calculations. First the hydrogenic limit model overestimates the charge distribution and the scattering atomic form factor. All results found in this present work can be easily extended to the helium like ions. The accuracy of the calculations made in this work may be substantially improved by increasing the size of the base of the test wave functions. This will have as a consequence, the lengthening of the calculation time.

\section{References}

[1] Hiroyuki Nakashima and Hiroshi Nakatsuji, (2007) Solving the Schrödinger equation for helium atom and its isoelectronic ions with the free iterative complement interaction (ICI) method, The Journal of Chemical Physics 127, 224104. 
[2] Scherer P. O. J. (2017) Variational Methods for Quantum Systems. In: Computational Physics. Graduate Texts in Physics. Springer, Cham pp 575-603.

[3] Lucjan Piela, (2014) Two Fundamental Approximate Methods. Ideas of Quantum Chemistry, Second Edition, Elsevier, pp 231-256.

[4] A. K. Roy, (2013) Studies on some exponential - screened coulomb potentials. International Journal of Quantum Chemistry 113, 1503.

[5] Arka Bhattacharya, M. Z. M. Kamali, Arijit Ghoshal and K. Ratnavelu, (2013) Physics of Plasmas 20, 083514.

[6] Neetik Mukherjee and Amlan K. Roy, (2019) Quantum mechanical virial-like theorem for confined quantum systems. Phys. Rev. A 99, 022123.

[7] K. D. Sen, Jacob Katriel, H. E. Montgomery, (2018) A comparative study of two-electron systems with screened Coulomb potentials. Annals of Physics 397, pp 192-212.

[8] Li Guang Jiao and Yew Kam Ho, (2014) Calculation of screened Coulomb potential matrices and its application to $\mathrm{He}$ bound and resonant states. Physical Review A 90, 012521.

[9] Freund, David E. and Huxtable, Barton D. and Morgan, John D. (1984) Variational calculations on the helium isoelectronic sequence. Phys. Rev. A, 29 (2): 980-982.

[10] Thakkar, Ajit J. and Koga, Toshikatsu. (1994) Ground-state energies for the helium isoelectronic series. Phys. Rev. A, 50 (1): 854-856.

[11] G. W. F. Drake. (1996) Atomic, molecular and optical physics handbook. AIP Press, Woodbury, NY\}, pp 154-171.

[12] Accad, Y. and Pekeris, C. L. and Schiff, B. (1975) Twoelectron $S$ and $P$ term values with smooth $Z$ dependence. Phys. Rev. A, 11 (4): 1479-1481.
[13] Green, Louis C. and Mulder, Marjorie M. and Milner, Paul C. (1953) Correlation Energy in the Ground State of He I, Phys. Rev. 91 (1): 35-39.

[14] Chandrasekhar, S. and Herzberg, G. (1955) Energies of the Ground States of He, $\mathrm{Li}^{+}$, and $\mathrm{O}^{6+}$. Phys. Rev. 98 (4): 1050 1054 .

[15] Hart, J. F. and Herzberg, G. (1957) Twenty-Parameter Eigen functions and Energy Values of the Ground States of $\mathrm{He}$ and He-Like Ions. Phys. Rev. 106 (1): 79-82.

[16] S Bhattacharyya and A Bhattacharyya and B Talukdar and N C Deb, (1996) Analytical approach to the helium-atom ground state using correlated wave functions. Journal of Physics B: Atomic, Molecular and Optical Physics 29 (5): L147.

[17] Hans A. Bethe and Edwin E. Salpeter, (1957) Quantum mechanics of one and two electron atoms. Springer Berlin pp 155.

[18] Korobov and Vladimir I. (2004) Bethe logarithm for the helium atom. Phys. Rev. A, 69 (5): 054501.

[19] Kar, S. and Ho, Y. K. (2006), Bound states of helium atom in dense plasmas. Int. J. Quantum Chem., 106: 814-822.

[20] Pekeris, C. L. (1959) $1^{1} S$ and $2^{3} S$ States of Helium. Phys. Rev. 115 (5): 1216-1221.

[21] Frankowski, K. and Pekeris, C. L. (1966) Logarithmic Terms in the Wave Functions of the Ground State of Two-Electron Atoms. Phys. Rev., 146 (1): 46-49.

[22] Frankowski, K. (1967) Logarithmic Terms in the Wave Functions of the $2^{1} S$ and $2^{3} S$ States of Two-Electron Atoms. Phys. Rev. 160 pp 1-3. 